\title{
Stent graft-induced new entry tear (SINE): Intentional and NOT
}

\author{
G. Chad Hughes, MD
}

\footnotetext{
$\overline{\text { From Department }}$ of Surgery, Division of Cardiovascular and Thoracic Surgery, Duke University Medical Center, Durham, NC.

Read at the 2018 American Association for Thoracic Surgery Aortic Symposium, New York, New York, April 2627, 2018.

Received for publication June 4, 2018; revisions received Oct 1, 2018; accepted for publication Oct 5, 2018. Address for reprints: G. Chad Hughes, MD, Center for Aortic Disease, Center for Structural Heart Disease, Duke University Medical Center, Division of Cardiovascular and Thoracic Surgery, Department of Surgery, Box 3051, Durham, NC 27710 (E-mail: gchad.hughes@ duke.edu).

J Thorac Cardiovasc Surg 2019;157:101-6

$0022-5223 / \$ 36.00$

Copyright (C) 2018 by The American Association for Thoracic Surgery

https://doi.org/10.1016/j.jtcvs.2018.10.060
}

The term stent graft-induced new entry (SINE) was originally defined by Dong and colleagues ${ }^{1}$ in 2010 as "a new tear caused by the stent graft itself, excluding those created by natural disease progression or any iatrogenic injury from the endovascular manipulation." Although the term SINE was originally coined in 2010, the issue of stent graft-induced intimal injury was recognized a decade earlier in a report by Ninomiya and colleagues, ${ }^{2}$ who described the development of a new "ulcer-like projection" at the distal end of a thoracic stent graft 14 months after implantation in a patient treated for chronic type $\mathrm{B}$ dissection (Figure E1). Since these early reports, a large amount of data has been published regarding the incidence, etiology, and outcomes of SINE after thoracic endovascular aortic repair (TEVAR), which will be reviewed in this manuscript. In addition, more recently, some authors have intentionally created intimal tears to allow the stent graft to approximate to the outer aortic wall with the goal of achieving more extensive aortic remodeling, and the limited data available regarding these "intentional SINE" techniques will be briefly reviewed as well.

\section{SINE INCIDENCE}

In their sentinel report in 2010, Dong and colleagues ${ }^{1}$ noted that the occurrence of SINE is most common after TEVAR performed for an aortic dissection indication. The authors described both proximal SINE, leading to retrograde type A dissection or proximal pseudoaneurysm formation, and distal SINE, resulting in false lumen pressurization and expansion. The reported incidence of SINE after TEVAR varies but may be as high as $25 \%$, , with distal SINE being more common and representing up to $80 \%$ or more of SINE in some series. ${ }^{4}$ Further, the complication of distal SINE has been increasingly reported, with multiple publications in the literature since $2010 .^{5-12}$ SINE are typically delayed in occurrence, with most developing approximately 12 to 36 months post-TEVAR. ${ }^{5-8,10}$ They are usually asymptomatic and

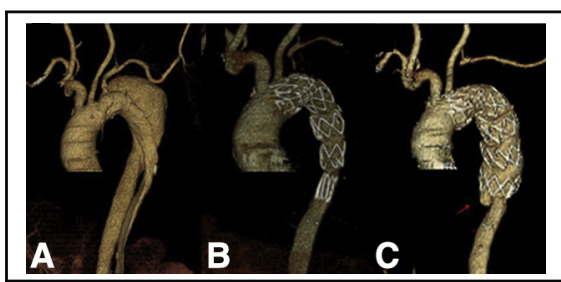

Serial CTA images demonstrating development of distal SINE. Modified with permission from Jang and colleagues.

\section{Central Message}

Stent graft-induced new entry is an increasingly recognized complication of thoracic endovascular repair for chronic dissection that may lead to therapeutic failure.

\section{Perspective}

Stent graft-induced new entry (SINE) is an increasingly recognized complication of TEVAR for chronic dissection that may lead to therapeutic failure. A "distal-first" deployment sequence appears effective in preventing SINE. Even with SINE avoidance, however, aortic growth may still occur. Techniques involving "intentional" SINE creation to obliterate all false lumen flow are in development.

See Editorial Commentary page 107.

discovered on routine postoperative surveillance imaging, ${ }^{10-12}$ a point that highlights the need for lifelong imaging surveillance follow-up in this population. Reintervention is usually required due to progressive aortic enlargement, ${ }^{12}$ which most commonly entails open surgery in the case of the less frequent proximal SINE (Figure 1) and distal extension TEVAR for distal SINE SI $^{3,4}$ (Figure E2; Table 1).

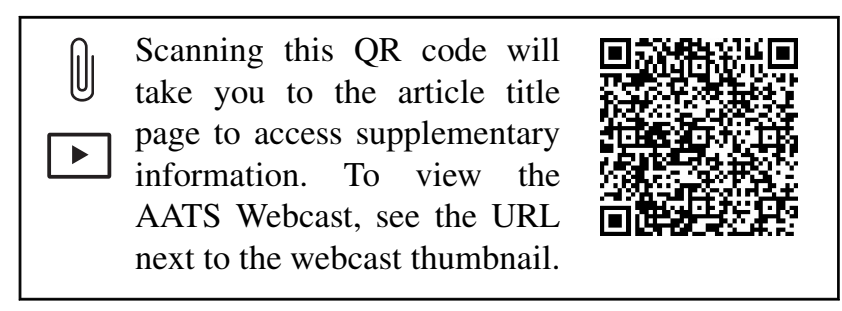




\section{Abbreviations and Acronyms}

SINE = stent graft-induced new entry

TEVAR $=$ thoracic endovascular aortic repair

\section{SINE RISK FACTORS AND ETIOLOGY}

A number of risk factors have been associated with the occurrence of SINE (Table 1). Foremost, distal SINE appears to be more common after TEVAR performed for chronic type B aortic dissection. ${ }^{4,6,7}$ The morphology of the dissected aorta changes over time, with the intimal flap thickening and becoming less mobile. ${ }^{13}$ As such, the dissected intimal flap cannot reapproximate to the outer aortic wall after TEVAR in the chronic setting, leading to persistent radial force exerted by the distal compressed stent graft, which may lead to SINE at the distal end of the device over time (Figure E3). ${ }^{3,6}$ Conversely, after TEVAR for acute dissection, the intimal flap is highly mobile, thereby allowing the distal end of the stent graft to fully expand such that the dissection flap may return to its previous anatomic position adjacent to the outer aortic wall.

The most important risk factor for distal SINE appears to be excessive oversizing of the distal stent graft relative to the smaller true lumen. ${ }^{3,5}$ This is mainly applicable to the chronic setting, where the compressed true lumen assumes a crescent shape that can make sizing for device selection more challenging. Previous work ${ }^{14}$ has demonstrated that the diameter of the true lumen in the distal thoracic aorta of patients with chronic dissection is approximately one third of the normal diameter in healthy controls, and therefore when endografts are sized only to the proximal nondissected true lumen in the aortic arch, as has been the standard for acute dissection, this results in an endograft that is markedly oversized $(>60 \%)$ in comparison with the distal true lumen.,

This marked oversizing leads to greater radial forces that can then induce intimomedial membrane disruption, as the distal landing zone is entirely within dissected aorta and the
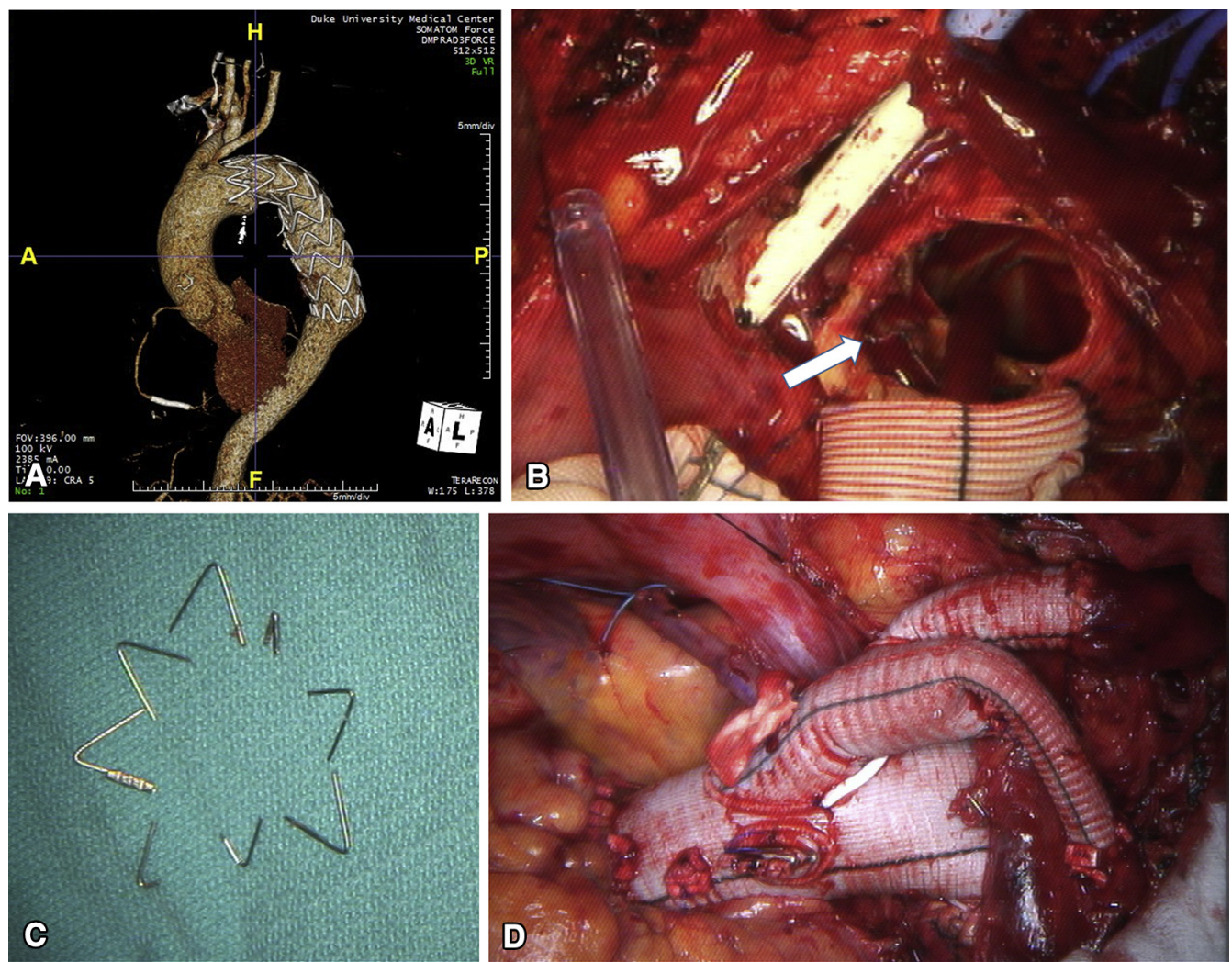

FIGURE 1. A, Preoperative 3-dimensional computed tomography angiography reconstruction image demonstrating the proximal bare springs of a thoracic endograft eroding into the wall of the aortic arch with the development of a proximal SINE/arch pseudoaneurysm (arrow). The device was placed for an acute complicated type B dissection 7 years earlier. Note the "spring back" apparent in the device configuration, similar to that described in Figure 2, with the early development of a distal SINE/pseudoaneurysm as well. B, Intraoperative photograph from the same patient showing the open aortic arch with bare springs embedded into the aortic wall (arrow). C, The arch was resected up to the level of the proximal end of the endograft and the proximal bare springs removed from the device. D, The arch was then replaced with a branched Dacron graft with the distal arch anastomosis incorporating the aortic wall and proximal end of the old endograft. 
TABLE 1. Subtypes, treatment options, and risk factors for stent graft-induced new entry tear after thoracic endovascular aortic repair

\begin{tabular}{ll}
\hline Subtype & Treatment options \\
\hline Proximal & Open repair \\
Distal & Distal TEVAR extension \\
& Open repair \\
\hline
\end{tabular}

\section{Risk factors}

TEVAR for chronic dissection

Excessive oversizing of distal stent graft relative to smaller true lumen

Connective tissue disorder

Shorter length $(<145 \mathrm{~mm})$ stent grafts

Proximal to distal stent graft implantation sequence in chronic dissection

TEVAR, Thoracic endovascular aortic repair.

fragile dissection membrane is more prone to injury. ${ }^{5}$ This may be especially problematic in patients with connective tissue disorders in whom the aorta has pathologic fragility even in the absence of previous dissection, and several studies have suggested the incidence of SINE to be greater in patients with Marfan syndrome. ${ }^{1,7}$ For example, in their sentinel report in 2010, Deng and colleagues ${ }^{1}$ noted a 10-fold greater incidence of SINE in patients with Marfan as compared with patients without Marfan syndrome after TEVAR for type B dissection and suggested trying to avoid the use of TEVAR in this population. Likewise, in a systematic review of the results of TEVAR for type B dissection in patients with Marfan syndrome, Pacini and colleagues ${ }^{15}$ noted a substantial risk of surgical conversion and death at midterm follow-up and suggested caution against the routine use of endovascular stent grafting in patients with Marfan syndrome.

Self-expanding TEVAR grafts have a tendency to "spring back" to their initial straight status if passively bent, such as when placed across the aortic arch
(Figure 2). ${ }^{1,6}$ This spring-back strength may be especially prominent in devices with longitudinal connecting bars that prevent twisting and kinking but sacrifice flexibility. ${ }^{1}$ This spring-back force on the distal end of the stent graft may then lead to distal SINE. Li and colleagues ${ }^{6}$ demonstrated that stent grafts with a length $<145 \mathrm{~mm}$ were associated with a greater risk of distal SINE, potentially due to the leverage effect of the lesser curve of the arch on the device, as shorter devices have greater spring-back force on their distal end.

Although one might hypothesize that the risk of SINE should vary based on device type, eg, stainless-steel, nitinol, presence or absence of connecting bar, etc, SINEs have been observed with all devices, and there is insufficient evidence in the literature to definitively suggest superiority of one device over another with regard to SINE incidence at the current time. ${ }^{4}$ Likewise, the currently available TEVAR devices also vary in the radial forces they exert on the aortic wall, ${ }^{16}$ and this characteristic of the individual devices may also contribute to SINE occurrence. However, only very limited data are available on this topic, ${ }^{16}$ and additional work examining all commercially available devices and sizes is needed to further elucidate the potential impact of this aspect of device design on SINE occurrence.

\section{SINE PREVENTION}

SINE prevention is important, as their occurrence may lead to pseudoaneurysm formation or retrograde dissection proximally and false lumen repressurization distally, both of which result in therapeutic failure. ${ }^{9,12,17}$ As SINE are most common at the distal end of a thoracic endograft implanted in the setting of chronic type B dissection, the following are means to minimize their occurrence. Most importantly, and unlike what has traditionally been done when sizing devices for acute dissection (where the device size is chosen based on the diameter of
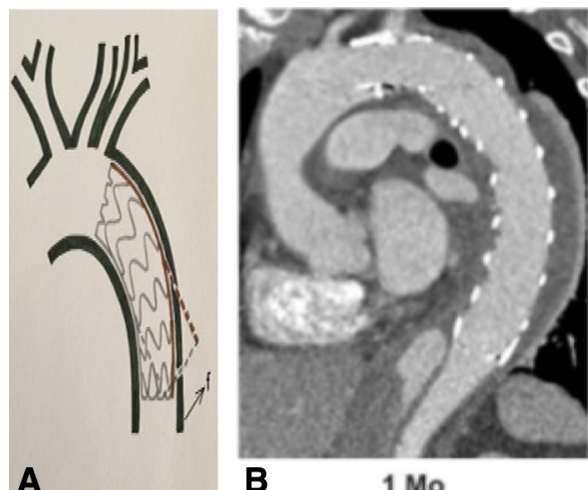

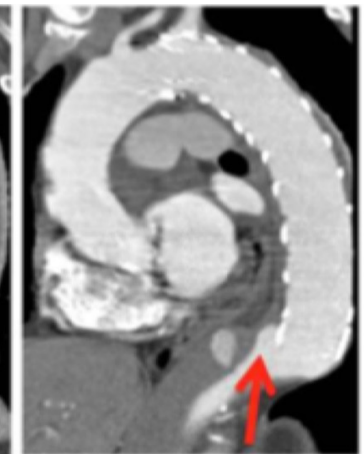

$3 \mathrm{Mo}$

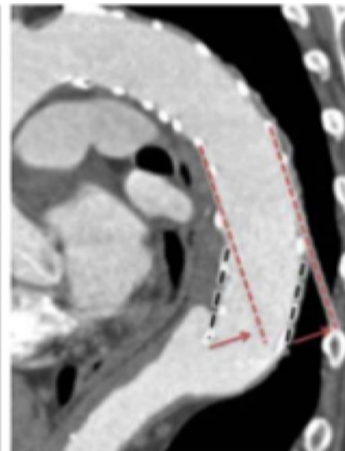

16 Mo

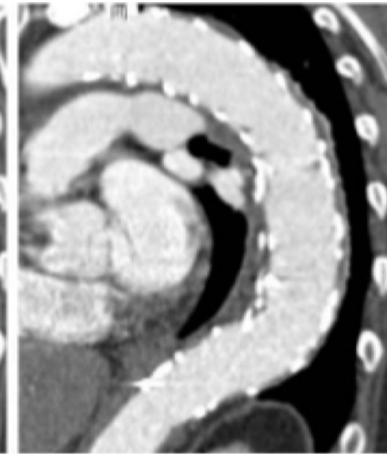

after Re-TEVAR

FIGURE 2. A, Drawing and B, serial CTA images illustrating the tendency of self-expanding stent grafts to "spring back" to their initial straight status if passively bent, such as when placed across the aortic arch. In the CTA images, this elastic recoil led to a distal SINE (large arrow, second panel) with thoracic aneurysm expansion requiring distal TEVAR reintervention. TEVAR, Thoracic endovascular aortic repair. A, modified from Li and colleagues ${ }^{6}$ with permission. B, reproduced from Jánosi and colleagues ${ }^{10}$ with permission. 


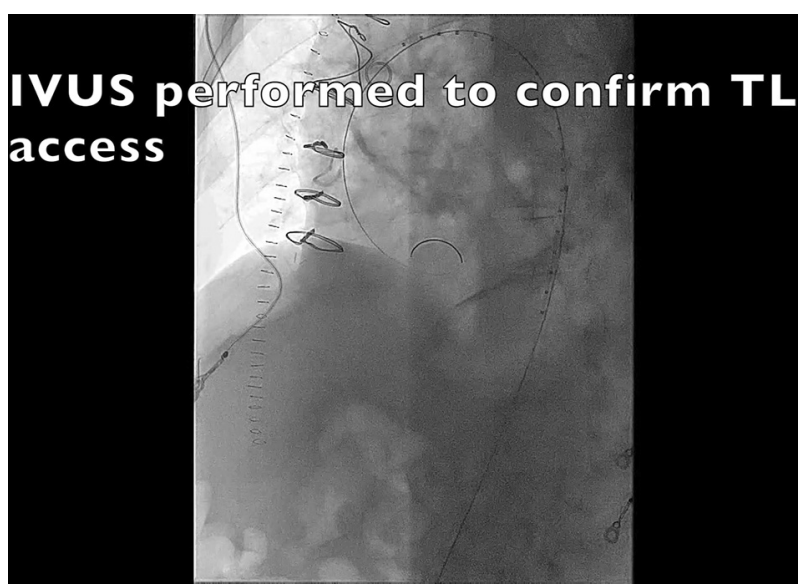

VIDEO 1. The "distal-first" implantation technique as used by our group for thoracic endovascular aortic repair (TEVAR) for chronic dissection. An 84-year-old patient is shown who presented with a large distal arch aneurysm secondary to chronic residual dissection 8 months after previous supracoronary ascending aorta and hemiarch replacement for acute type A dissection at another institution. He underwent first-stage redo-sternotomy for 3-vessel arch debranching, and the video shows his second-stage completion TEVAR procedure performed several days later during the same hospital stay. The beginning of the video shows an intravascular ultrasound (IVUS) probe being used to interrogate the entire aorta to confirm true lumen wire location throughout (IVUS images not shown). The devices are then deployed from distal to proximal in the true lumen, beginning just above the celiac axis with a $31 / 26 \mathrm{~mm} \times 10 \mathrm{~cm}$ conformable tapered device deployed distally, followed by a $34 \mathrm{~mm} \times 20 \mathrm{~cm}$ device more proximally. Following a proximal marker arteriogram to roadmap the origin the arch debranching graft and proximal landing zone, a $40 \mathrm{~mm} \times 20 \mathrm{~cm}$ device is deployed. The completion angiogram demonstrates no endoleak, and the 1-month follow-up 3-dimensional reconstructed computed tomography angiography image demonstrates a good result with a thrombosed aneurysm sac and thoracic false lumen. The patient continues to do well now nearly 1 year after his 2 -stage type I hybrid arch repair with no endoleak and significant shrinkage of his distal arch aneurysm sac. Video available at: https://www.jtcvs.org/article/ S0022-5223(18)32821-6/fulltext.

nondissected proximal landing zone in the arch), device selection must take into account measurements of the distal landing zone as well. ${ }^{18}$ Specifically, as the distal device will land entirely within the true lumen of the chronically dissected aorta, the true lumen is measured using curved planar reformats and a mean diameter of the true lumen obtained (Figure E4). The device is then chosen such that there is minimal oversizing of the distal landing zone. Our group ${ }^{9,18}$ has evolved to using a distal to proximal endograft implantation sequence, as originally proposed by Weng and colleagues, ${ }^{3}$ with tapered, low radial force devices. The distal device is most commonly a 31/26-mm diameter tapered device, with the devices then deployed from distal to proximal with the most proximal device diameter typically in the $37-$ to $45-\mathrm{mm}$ range as appropriate for the nondissected proximal landing zone (Video 1). Most often, 3 devices are required to span this diameter range. This results in a $15-$ to $20-\mathrm{mm}$ taper from distal to proximal with regard to device diameter, but using this technique we have completely avoided SINE occurrence. Recent work by Chen and colleagues ${ }^{19}$ has validated this technique, with a marked reduction in SINE occurrence when a distal first sequence of stent graft deployment was used in chronic type B dissection.

Alternatively, another technique involves deploying a restrictive bare metal stent in the true lumen at the level of the intended distal landing zone of the endograft, before the introduction and deployment of the stent graft, such that the bare metal stent limits the extent to which the endograft may expand. This slightly more complex technique has been shown to limit the occurrence of distal SINE, although limited data exist. ${ }^{11}$ Finally, the Candy-Plug technique, which uses a double-tapered tubular stent-graft deployed intentionally within the distal false lumen adjacent to the distal end of a thoracic stent graft placed in the true lumen at the level of the celiac axis and then occluded with either an occlusion plug or occluder device, also has been used to prevent distal SINE. ${ }^{20}$ The theory is that the distal alignment of the true and false lumen stent grafts protects the intimomedial membrane from the radial forces exerted by the true lumen device so as to prevent distal SINE.

\section{"INTENTIONAL" SINE}

A recent systematic review highlighted the evidence that, based on the available data, TEVAR in patients with aortic dissection does not appear to reliably prevent aneurysmal degeneration of the thoracic or abdominal aorta. This is true whether TEVAR is performed in the acute or chronic phase. ${ }^{21}$ The major reason for this finding is that persistent false lumen perfusion via distal entry tears is common after TEVAR for dissection (Figure E5), and this may occur despite seal of the proximal entry tear. ${ }^{20}$ As such, a number of creative techniques have been proposed to intentionally fracture the intimomedial membrane so as to allow the stent graft to approximate to the outer aortic wall with the goal of achieving more extensive aortic remodeling. All of the techniques have been reported only in small series of highly selected patients, and additional data are needed before any can be recommended. Likewise, although persistent false lumen perfusion via distal entry tears is common after TEVAR for dissection, this does not uniformly result in aortic expansion, ${ }^{9}$ and therefore the use of these adjunctive techniques in all patients is likely unnecessary and would result in overtreatment of some. This highlights the current critical need for additional research delineating those dissection anatomies best treated with TEVAR alone versus TEVAR plus adjunctive false lumen-obliterating techniques. 

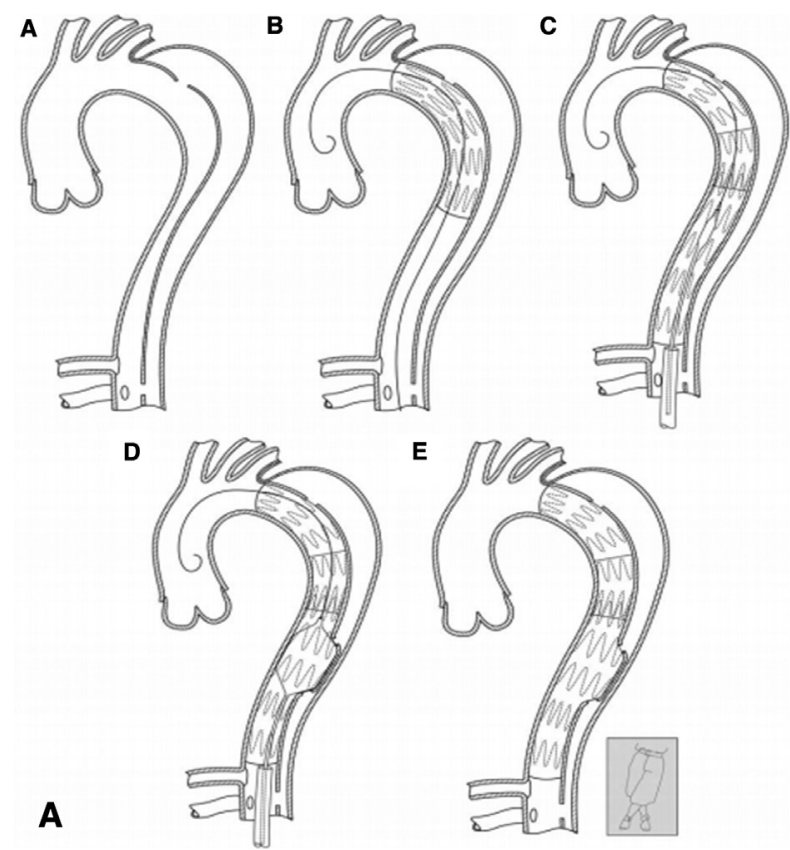

A

FIGURE 3. A, The "Knickerbocker technique" for treating chronic typ
aorta then paved down to the celiac axis with endografts in the true lume
dissection membrane and allow the distal endograft to expand outward to re
more proximal thoracic false lumen. Reproduced from Kölbel and collea
relamination technique for treating type B aortic dissection. A covered en
entry tear with bare metal stents then deployed distally over the visceral
then balloon dilated to disrupt the intimal flap and obliterate the distal fa
The "Knickerbocker technique" consists of proximal TE VAR to cover the primary entry tear with a second oversized endograft deployed more distally. A large compliant balloon is then inflated within the mid-portion of this larger endograft to rupture the dissection membrane and allow the endograft to expand to the outer aortic wall, thereby preventing back flow into the more proximal thoracic false lumen ${ }^{22}$ (Figure 3). The so-called stent-assisted balloon-induced intimal disruption and relamination technique was first reported in $2014^{23}$ and involves proximal TEVAR to cover the primary entry tear with distal extension bare metal stents along the length of the distal flap followed by ballooninduced disruption of the distal intimal flap beginning in the distal third of the stent-graft and extending along the length of the uncovered dissection stents so as to recreate a single flow channel throughout the thoracoabdominal aorta (Figure 3). Finally, some authors ${ }^{24}$ have used various techniques to create endovascular longitudinal fenestrations in the intimal flap, so-called "intimal flap septostomy," again with the goal of creating a distal landing zone in which the stent-graft may be apposed to the outer aortic wall.

In summary, SINE is an increasingly reported complication of TEVAR for chronic type B dissection that may lead to therapeutic failure or need for reintervention. A number of techniques have been proposed to limit its occurrence,
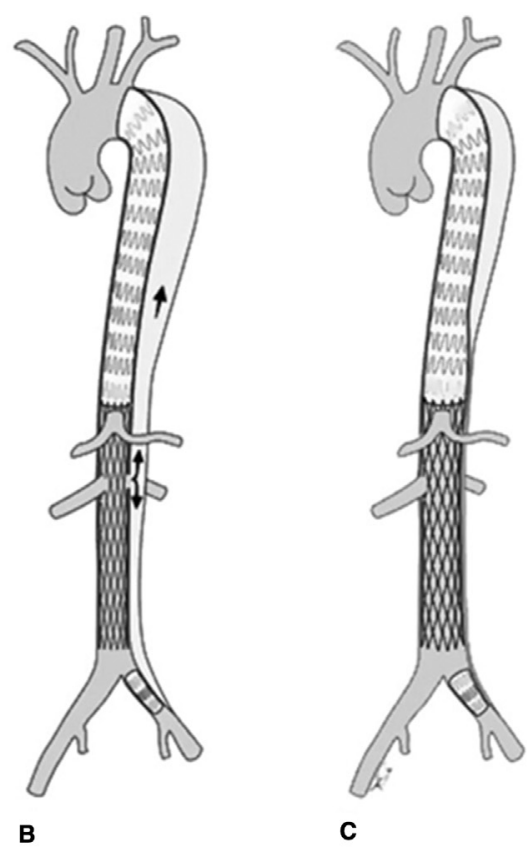

c

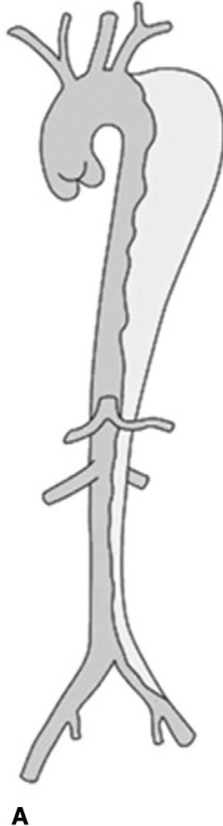

B dissection with aneurysm whereby the proximal entry tear is covered and the 


\section{Conflict of Interest Statement}

Author has nothing to disclose with regard to commercial support.

\section{References}

1. Dong Z, Fu W, Wang Y, Wang C, Yan Z, Guo D, et al. Stent graft-induced new entry after endovascular repair for Stanford type B aortic dissection. J Vasc Surg. 2010;52:1450-8.

2. Ninomiya M, Takamoto S, Kotsuka Y, Kubota H. Stent-graft-induced intimal injury one year after surgery. J Thorac Cardiovasc Surg. 2002; 123:371-2.

3. Weng S-H, Weng C-F, Chen W-Y, Huang C-Y, Chen I-M, Chen C-K, et al. Reintervention for distal stent graft-induced new entry after endovascular repair with a stainless steel-based device in aortic dissection. J Vasc Surg. 2013;57: 64-71.

4. Jang H, Kim M-D, Kim GM, Won JY, Ko Y-G, Choi D, et al. Risk factors for stent graft-induced new entry after thoracic endovascular aortic repair for Stanford type B aortic dissection. J Vasc Surg. 2017;65:676-85.

5. Huang C-Y, Weng S-H, Weng C-F, Chen W-Y, Chen I-M, Hsu C-P, et al. Factors predictive of distal stent graft-induced new entry after hybrid arch elephant trunk repair with stainless steel-based device in aortic dissection. J Thorac Cardiovasc Surg. 2013;146:623-30.

6. Li Q, Wang L-F, Ma W-G, Xu S-D, Zheng J, Xing X-Y, et al. Risk factors for distal stent graft-induced new entry following endovascular repair of type B aortic dissection. J Thorac Dis. 2015;7:1907-16.

7. Pantaleo A, Jafrancesco G, Buia F, Leone A, Lovato L, Russo V, et al. Distal stent graft-induced new entry: an emerging complication of endovascular treatment in aortic dissection. Ann Thorac Surg. 2016;102:527-33.

8. Huang C-Y, Chen C-W, Chen P-L, Chen W-Y, Chen I-M, Hsu C-P, et al. Association between aortic remodeling and stent graft-induced new entry in extensive residual type A dissecting aortic aneurysm after hybrid arch repair. Ann Vasc Surg. 2016:31:60-9.

9. Hughes GC, Ganapathi AM, Ke enan JE, Englum BR, Hanna JM, Schechter MA, et al. Thoracic endovascular aortic repair for chronic DeBakey IIIlb aortic dissection. Ann Thorac Surg. 2014;98:2092-7.

10. Jánosi RA, Tsagakis K, Bettin M, Kahlert P, Horacek M, Al-Rashid F, et al. Thoracic aortic aneurysm expansion due to late distal stent graft-induced new entry. Catheter Cardiovasc Interv. 2015;85:E43-53.

11. Zhao Y, Yin H, Chen Y, Wang M, Zheng L, Li Z, et al. Restrictive bare stent prevents distal stent graft-induced new entry in endovascular repair of type B aortic dissection. J Vasc Surg. 2018;67:93-103.

12. Huang C-Y, Hsu H-L, Chen P-L, Chen I-M, Hsu C-P, Shih C-C. The impact of distal stent graft-induced new entry on aortic remodeling of chronic type B dissection. Ann Thorac Surg. 2018;105:785-93.
13. Peterss S, Mansour AM, Ross JA, Vaitkeviciute I, Charilaou P, Dumfarth J, et al. Changing pathology of the thoracic aorta from acute to chronic dissection. Literature review and insights. J Am Coll Cardiol. 2016;68:1054-65.

14. Xu SD, Huang FJ, Du JH, Li Y, Fan ZM, Yang JF, et al. A study of aortic dimension in type B aortic dissection. Interact Cardiovasc Thorac Surg. 2008; 7:244-8.

15. Pacini D, Parolari A, Berretta P, DiBartolomeo R, Alamanni F, Bavaria J. Endovascular treatment for type B dissection in Marfan syndrome: is it worthwhile? Ann Thorac Surg. 2013;95:737-49.

16. Matsumoto T, Inoue K, Tanaka S, Aoyagi Y, Matsubara Y, Matsuda D, et al. Radial forces of stents used in thoracic endovascular aortic repair and bare self-expanding nitinol stents measured ex vivo-rapid rescue for obstruction of the innominate artery using bare self-expanding nitinol stents. Vascular. 2017; 25:36-41.

17. Chen I-M, Chen P-L, Huang C-Y, Weng S-H, Chen W-Y, Shih C-C. Factors affecting optimal aortic remodeling after thoracic endovascular aortic repair of type B (IIIb) aortic dissection. Cardiovasc Intervent Radiol. 2017;40: 671-81.

18. Hughes GC, Andersen ND, McCann RL. Endovascular repair of chronic type B aortic dissection with aneurysmal degeneration. Op Tech Thorac Cardiovasc Surg. 2013;18:101-16.

19. Chen I-M, Huang C-Y, Wen S-H, Lin P-Y, Chen P-L, Chen W-Y, et al. Implantation sequence modification averts distal stent graft-induced new entry after endovascular repair of Stanford type B aortic dissection. J Vasc Surg. 2016; 64:281-8.

20. Rohlffs F, Tsilimparis N, Fiorucci B, Heidemann F, Debus ES, Kölbel T. The candy-plug technique: technical aspects and early results of a new endovascular method for false lumen occlusion in chronic aortic dissection. J Endovasc Ther. 2017;24:549-55.

21. Famularo M, Meyermann K, Lombardi JV. Aneurysmal degeneration of type B aortic dissections after thoracic endovascular aortic repair: a systematic review. J Vasc Surg. 2017;66:924-30.

22. Kölbel T, Carpenter SW, Lohrenz C, Tsilimparis N, Larena-Avellaneda A, Debus ES. Addressing persistent false lumen flow in chronic aortic dissection: the knickerbocker technique. J Endovasc Ther. 2014;21:117-22.

23. Hofferberth SC, Nixon IK, Boston RC, McLachlan CS, Mossop PJ. Stent-assisted balloon-induced intimal disruption and relamination in aortic dissection repair: the STABILISE concept. J Thorac Cardiovasc Surg. 2014; $147: 1240-5$.

24. Saito Y, Tani K, Taniguchi S, Fukuda I. Endovascular "intimal flap septostomy" for safe landing of a stent graft in an anastomotic pseudoaneurysm of chronic type B aortic dissection. EJVES Short Rep. 2017;37:5-7.

Key Words: aorta, surgery, dissection, endovascular, complication 


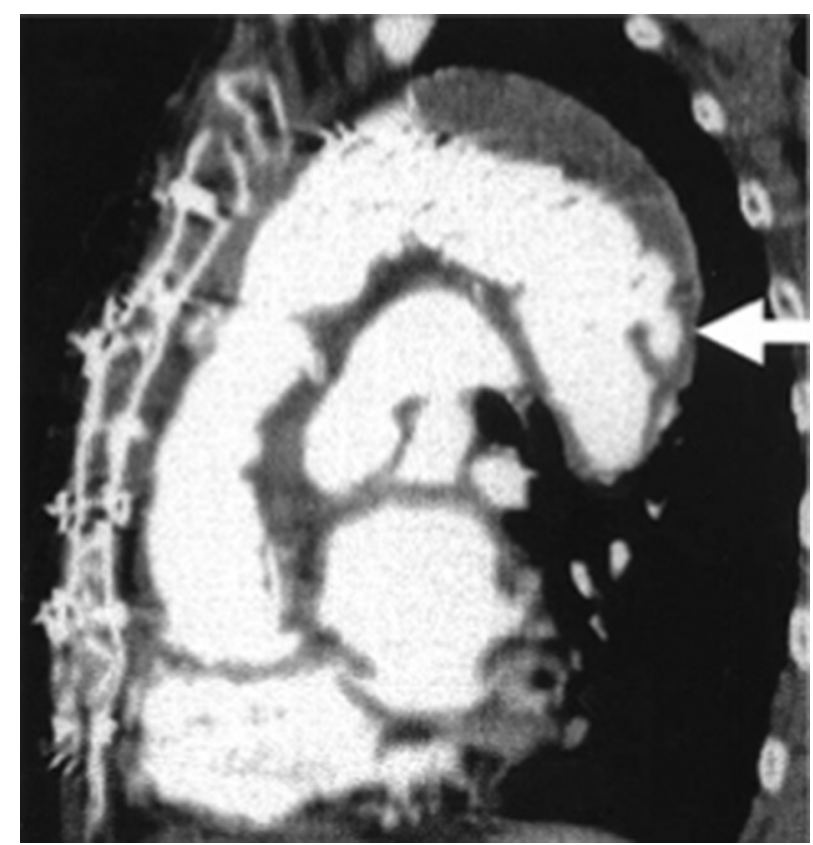

FIGURE E1. Sagittal reconstructed computed tomography angiography image from the sentinel report of stent graft-induced intimal injury published in 2002. ${ }^{2}$ The image demonstrates a new "ulcer-like projection" (arrow), which developed at the distal end of an early generation thoracic stent graft 14 months after implantation in a patient treated for chronic type B dissection. Reproduced from Ninomiya and colleagues ${ }^{2}$ with permission.
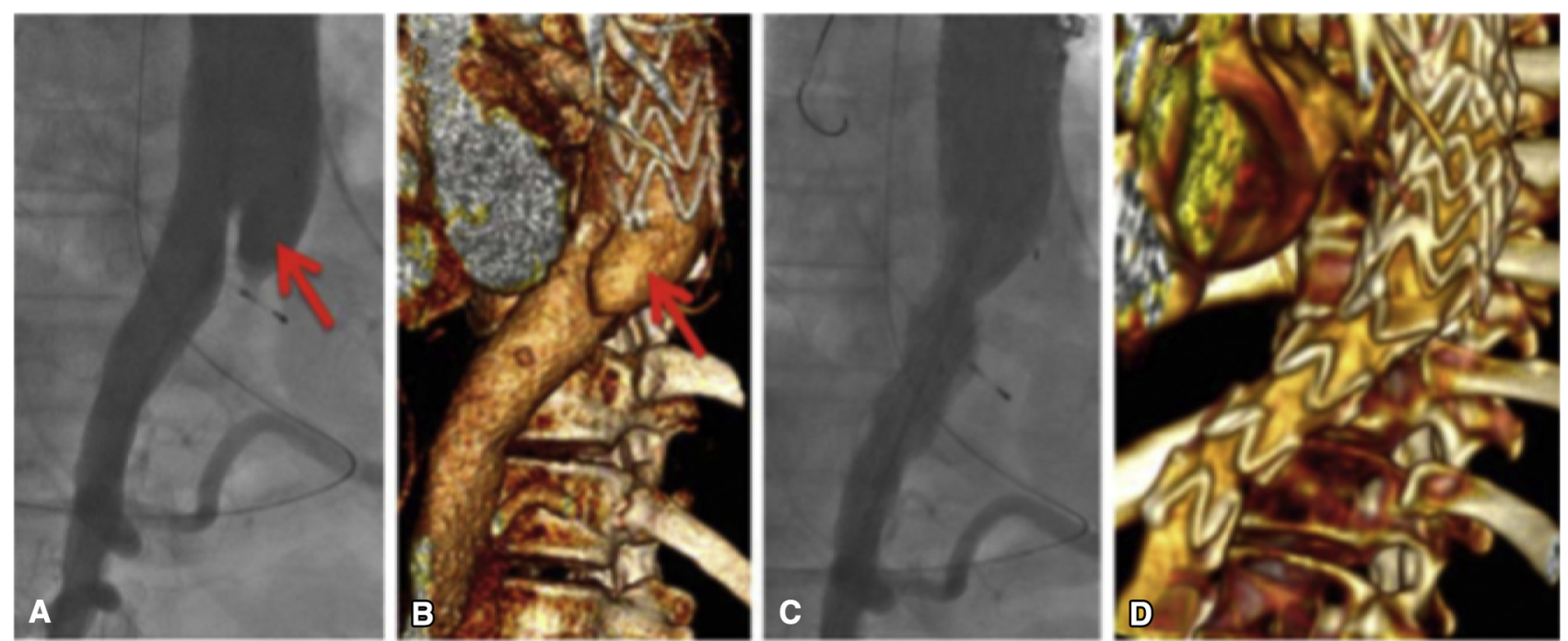

FIGURE E2. A, Angiogram and B, 3-dimensional reconstructed computed tomography angiography images demonstrating a distal stent graft-induced new entry (arrows), which was treated with distal extension thoracic endovascular aortic repair (C and D). Modified from reference Jánosi and colleagues ${ }^{10}$ with permission. 


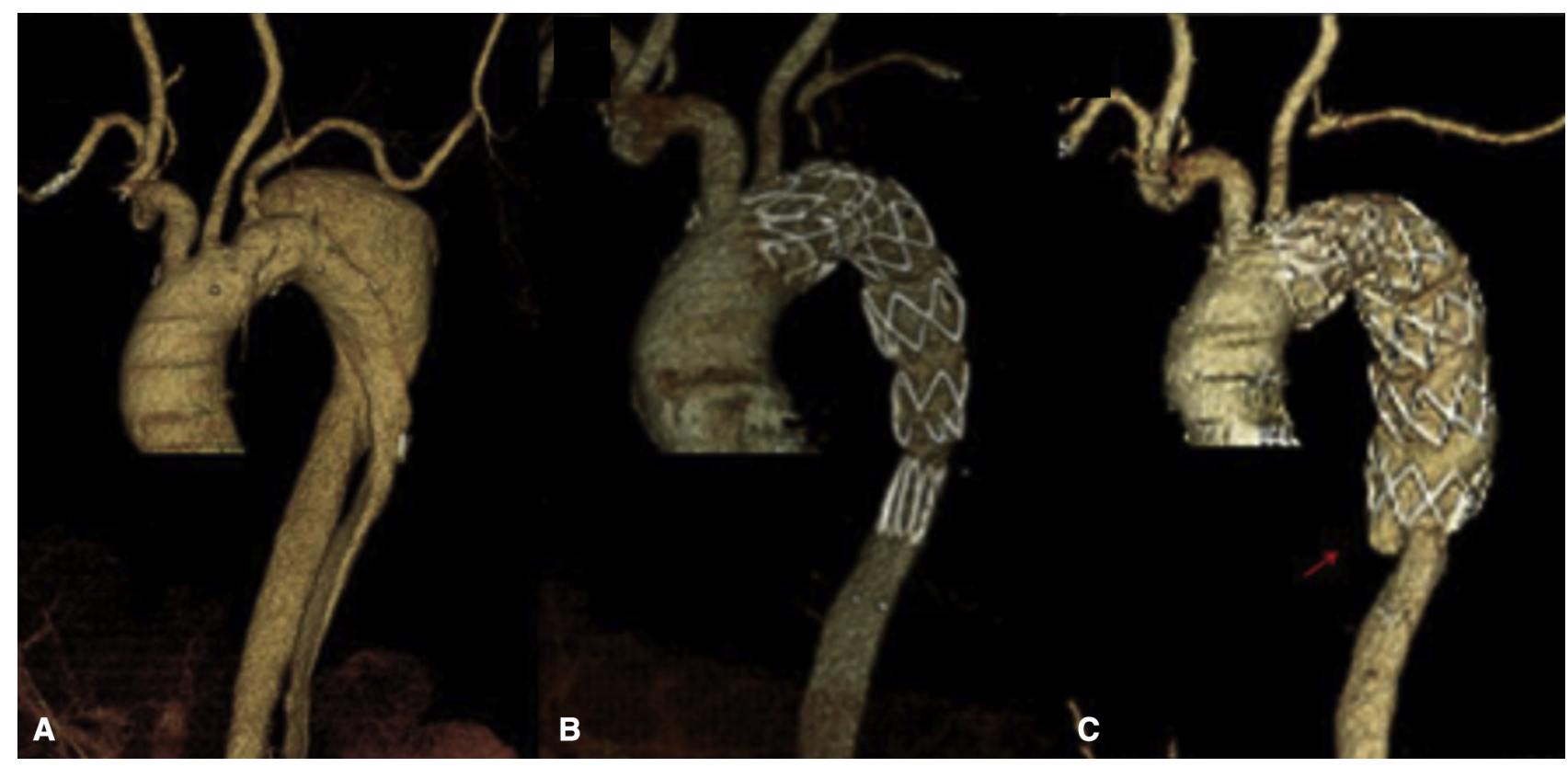

FIGURE E3. Serial 3-dimensional reconstructed computed tomography angiography reconstruction images demonstrating a distal compressed stent graft in the small true lumen after thoracic endovascular aortic repair for chronic dissection (A and B). C, Over time, the persistent radial force of the compressed endograft leads to distal stent graft-induced new entry (arrow). Modified from Jang and colleagues ${ }^{4}$ with permission.

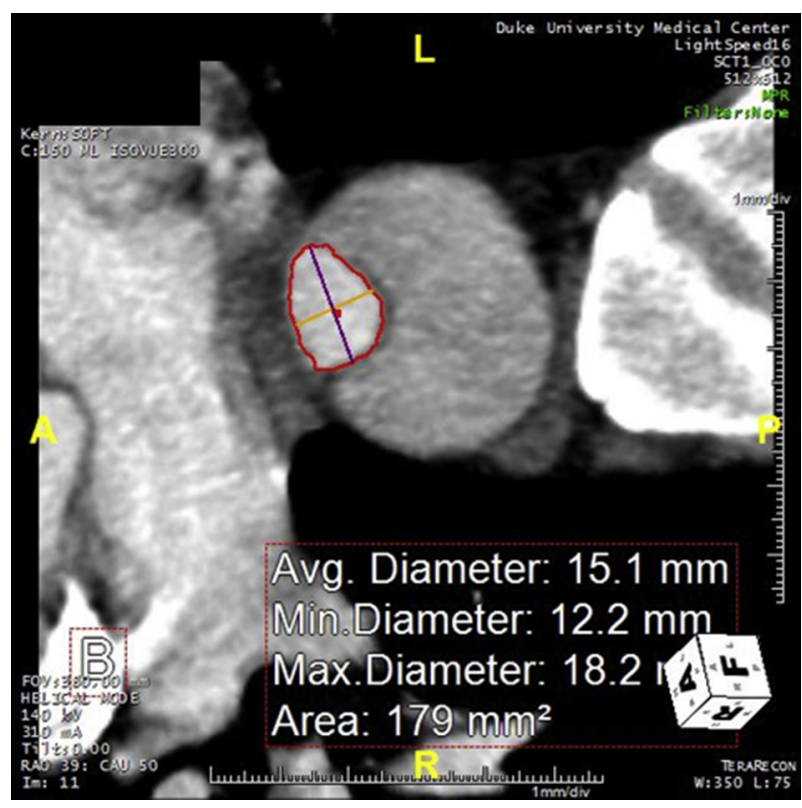

FIGURE E4. Curved planar reformat computed tomography angiography reconstruction measuring the mean diameter of the true lumen in the distal landing zone in the distal descending thoracic aorta. The distal thoracic endovascular aortic repair device is then chosen such that there is minimal oversizing of the proposed distal landing zone so as to prevent distal stent graft-induced new entry occurrence. Reproduced from Hughes and colleagues ${ }^{18}$ with permission. 


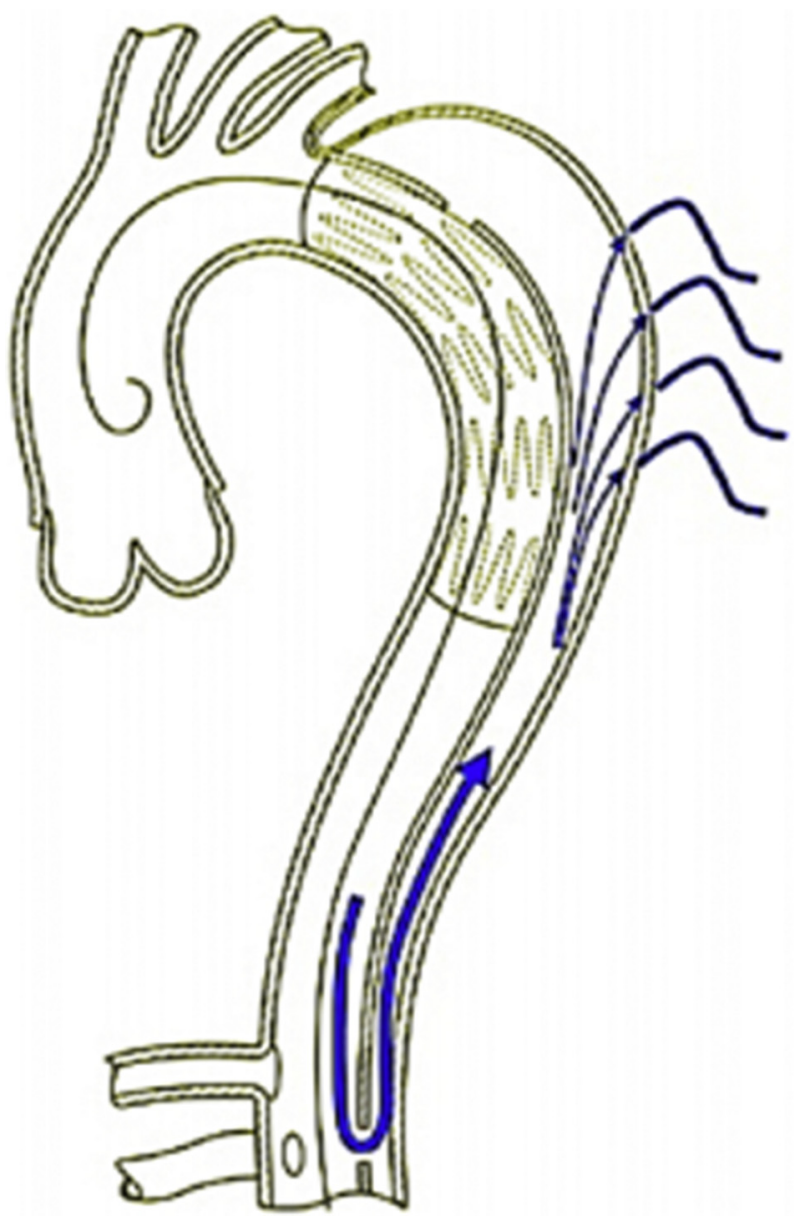

FIGURE E5. Concept of retrograde false lumen pressurization via downstream fenestrations. In most cases of DeBakey type IIIb aortic dissection, there are multiple downstream fenestrations that continue to provide flow to the false lumen despite adequate coverage of the proximal primary tear and even with avoidance of stent graft-induced new entry creation. These downstream re-entry tears are especially common in the visceral segment, where they are located at the prior ostia of branch vessels, which now arise from the false lumen such that a channel connecting the true and false lumens at this level exists. Retrograde flow from the abdominal aorta back up into the false lumen of the thoracic aorta is generally only seen when there are patent intercostal or bronchial arteries arising from the false lumen to allow continued flow. This may also be seen when the left subclavian artery either partially or fully arises from the false lumen to allow continued retrograde flow with false lumen pressurization. Reproduced from Rohlffs and colleagues ${ }^{20}$ with permission. 